Revue d'histoire de l'Amérique française

सQS REVUE D.HISTOIRE DE L'AMÉRIQUE FRANÇAISE

\title{
Les dépenses du roi au Canada à l'époque de Jean Talon (1665-1672)
}

\section{Roland Lamontagne}

Volume 13, numéro 3, décembre 1959

URI : https://id.erudit.org/iderudit/301986ar

DOI : https://doi.org/10.7202/301986ar

Aller au sommaire du numéro

Éditeur(s)

Institut d'histoire de l'Amérique française

ISSN

0035-2357 (imprimé)

1492-1383 (numérique)

Découvrir la revue

Citer cet article

Lamontagne, R. (1959). Les dépenses du roi au Canada à l'époque de Jean Talon (1665-1672). Revue d'histoire de l'Amérique française, 13(3), 335-343.

https://doi.org/10.7202/301986ar d'utilisation que vous pouvez consulter en ligne.

https://apropos.erudit.org/fr/usagers/politique-dutilisation/ 


\section{LES DÉPENSES DU ROI AU CANADA À L'ÉPOQUE DE JEAN TALON *}

(1665-1672)

Cette brève étude a pour but de grouper chronologiquement les données financières de la politique coloniale française lors de la double période d'administration de l'intendant Talon.

La documentation indiquée dans l'article intitulé «Essai sur les finances canadiennes $(1700-1750) \gg$ de Guy Frégault ${ }^{1}$ et l'Inventaire Provisoire ${ }^{2}$ des documents français aux Archives Publiques du Canada ont facilité les recherches.

En 1665, Colbert remettait à l'intendant un état du revenu de la colonie et le compte des dépenses effectuées précédemment. Il enjoignait à l'intendant de vérifier de façon précise ces états financiers. "Comme il [Talon] pourra, estant sur les lieux, en tirer encore plus d'éclaircissement, l'intention du Roy est qu'il tasche d'entrer si avant dans cette matière qu'il connaisse avec certitude jusqu'au dernier sol à quoi ce revenu monte effectivement...»

Une note marginale de Talon signale que l'«administration des deniers publics dans les temps precedens, sera examinée

* Cet article est extrait d'un ouvrage intitulé, Jean Talon et le développement systématique du Canada, en cours de publication au Editions Fides.

L'auteur tient à remercier M. Pierre Brunet, Adjoint de l'Archiviste du Canada, qui lui a fait parvenir des copies microfilmées négatives des extraits des Archives des Colonies:

Série B 1 : 115 - 145. - Série B $2: 40$ - 55. - Série B $3: 33$ - 47. Série C 11 A, $113-1: 12-19$ - Série F 2 A, carton $13: 70-85$. Ibid., carton $15: 14-65$. - Ibid., carton $17: 2-27,67$. - Les copies microfilmées F2 et F3 de la Série AC, C 11 A, volumes 2 et 3, ont été consultées aux Archives Publiques du Canada. Grâce au service de prêt, elles ont été étudiées à la Bibliothèque de l'Université de Montréal. 308.

1 Revue d'Histoire de l'Amérique Française, XII (Décembre, 1958) : $1952)$.

2 Archives Publiques du Canada. Fonds des Manuscrits, 1 (Ottawa, 
a fond apres que les troupes auront reçeu le secours que je leur dois pour tout l'hiver ». ${ }^{3}$ L'intendant indique à Colbert les difficultés de financement de la colonie. Cet extrait d'un mémoire du 13 novembre 1666 nous fait comprendre le bien-fondé des réclamations de Talon.

Mais je vous supplie très humblement de faire ordonner que du fonds fait pour les troupes qui servent icy Le Tresorier en remette un tiers sur mon billet portant promesse d'en tenir compte et de luy en fournir descharges Vallables parce que les vaisseaux n'arrivant icy qu'au mois de Juin et plus tard, ainsy que cette année en laquelle ils n'ont paru qu'au mois d'Aoust Je suis obligé pour soustenir en les attendant L'officier et Le soldat, et fournir aux autres despenses, d'emprunter de toute part, et rendre après à Paris et ailleurs ainsy que j'ay fait dans tout le cours de l'année présente, cela n'augmentera pas la despense du Roy, et me facilitera de beaucoup son service. ${ }^{4}$

Le 21 mai 1665, Talon mentionnait au ministre qu'il avait acquitté les frais d'appointements des officiers du Régiment de Carignan jusqu'au mois de janvier 1666 inclusivement. Il note que les vaisseaux de la métropole n'arriveront que vers la mijuillet 1666 et qu'il sera obligé de solder lui-même les dépenses militaires. Il s'exprime sans ambages: «je les auray infailliblement sur les bras durant prez de six mois et je seray cependant en un pays où il n'y a pas de resources et où l'industrie des gens est bientost épuisée $\gg .5$

Les dépenses de la colonie étaient prélevées sur le produit de la ferme et les fonds de la métropole. Le 5 avril 1667, Colbert recommandait à l'intendant d'établir à 36,000 livres les dépenses qui devaient être acquittées par le produit de la ferme du «droict qui se leve sur les Castors \& du dixiesme des Orignaux ». Il insiste sur la nécessité de pratiquer une économie rigoureuse: «bien entendu que vous prendrez grand soin d'en faire l'employ

3 Mémoire du roi pour servir d'instruction à Talon (27 mars 1665) avec notes de Talon, Rapport de l'Archiviste de la Province de Québec pour l'année 1930-1931, 15.

4 Talon à Colbert, RAPQ (1930-1931), 58.

5 Ibid., 27. 
avec une exacte oeconomie d'autant plus qu'avant cette concession ces charges du pays qui estoient prises sur le mesme fonds ne montoient pas a $20^{\mathrm{m} \varepsilon} \&$ depuis la concession a $29^{\mathrm{m}} \varepsilon$ estant la seule utilité que cette Compagnie peut tirer de la Colonie pour compenser tous les fraix differents qu'elle est obligée de faire ». ${ }^{6}$

La réponse de l'intendant à cette recommandation du ministre contient une approximation du coût d'administration de la colonie pour l'année $1664 .^{7}$

Produits de la ferme

Fonds du roi pour le secours extraordinaire du Canada ... $\underline{35,000}$ “ rapportées par Talon)

\section{6,500 livres}

(moyenne des données

$\overline{81,500}$ “

Les deniers publics provenant du «quart des pelleteries》 servent à acquitter les appointements des gouverneurs particuliers, les traitements des officiers de justice, les subventions aux institutions religieuses et à l'hôpital, les ouvrages et réparations ainsi que les dépenses diverses. L'intendant rappelle au ministre que ce droit du quart des pelleteries constitue «tout le fonds des deniers publics sur lequel s'empruntent les charges indispensables du pays, et dans les besoins pressans, les secours essentiels à son salut....». 8

A l'automne de 1665, Talon établissait un état des dépenses qui devaient être prélevées sur le fonds du pays; il signalait d'《autres dépenses qu'on ne peut prévoir et que le temps seul fera connoistre ». En 1665, ces frais d'administration prélevés sur le produit de la ferme se chiffraient à 54,000 livres. ${ }^{9}$ Ce relevé financier pour l'année 1665 ne tient pas compte des dépenses du roi assignées sur le fonds du Ministère de la Marine.

6 Ibid., 70.

7 «...en mille six cens soixante quatre courant à 65 on a consommé outre tout le produit de la ferme de 46.500 livres la somme de 30 ou $40 \mathrm{~m} £$ qui furent envoyés par sa Maté pour le secours extraordinaire du Canada ». Talon à Colbert, Québec, 27 octobre 1667, Ibid., 82.

8 Talon à Colbert, Québec, 4 octobre 1665, Ibid., 36.

9 « Estat des charges du pays de Canada le payement desqu.1les s'est tousjours fait des deniers publics provenant du droict du quart des pelleteries ». AC, C11A, copie microfilmée F2 des Archives Publiques du Canada, volume $2: 159-160$. 
Ces fonds de la métropole sont indiqués dans un mémoire concernant des frais d'administration pour les années 1665 et 1666 que l'on verra plus loin.

Le 13 novembre 1666, l'intendant remerciait Colbert du privilège de convertir une partie de ses appointements en denrées exemptes de frais de fret sur lesquelles il effectuait un profit. Il assurait le ministre qu'il en ferait bon usage. ${ }^{10}$ Il avait fait construire un vaisseau de 120 tonneaux. Le ministre se montrait satisfait de cette activité diligente de l'intendant qui donnait aux habitants de la colonie l'exemple d'un stimulant économique. ${ }^{11}$

Pour solder les dépenses de l'exercice financier de 1666, Talon a eu recours à la «mesme somme du produit de la ferme que le Conseil employoit les années précédentes, tant pour acquitter Les pensions, et charges Indispensables du pays, que pour les autres dépenses a faire pour Le service du Roy, et la conservation de la Colonie ... » ${ }^{12}$

Les dépenses du roi relatives à l'entretien du Régiment Carignan-Sallières au Canada s'élevaient à 233,074 livres pour l'année 1666. ${ }^{13}$

L'intendant trouve que l'administration de la colonie coûte cher; les frais sont si élevés qu'il craint d'être mal jugé au Ministère de la Marine: «Je devrois en cet endroit vous faire un destail des despenses auxquelles ce pays icy engage mais a la vérité Je n'ose tant j'ay de confusion de celles que j'y ay faites, et tant j'ay de crainte de ne paroistre pas bon oeconome du bien du Roy $\gg^{14}$

10 Talon à Colbert, Québec, 13 novembre 1666, RAPQ (1930-1931), 59. 11 Colbert à Talon, Saint-Germain-en-Laye, 5 avril 1667, Ibid., 70. 12 Talon à Colbert, 13 novembre 1666, Ibid., 53.

13 Un abrégé de cet état financier comporte les chapitres suivants: Payemens faicts par Monsieur le Tresorier de l'extraordre

Pour vivres

Habits et commodités

Fournitures pour les officiers .......................................... 40,061. 14

Pour ustanciles 70,084. 19

Somme $10,355 . \quad 1$

Pour ustanciles des Magasins 158,538. 17

Total de toutte la despense $74,535.15$ AC, C11A, 2 : $445-446$.

14 Talon à Colbert, Québec, 13 novembre 1666, RAPQ (1930-1931), 55. 
Le relevé des dépenses pour les années 1665 et 1666 s'appuie sur le «Mémoire des fonds faicts pour le paiement des Troupes et officiers majors passés au Canada depuis le premier janvier $1665 .{ }^{15}$

Cet état financier au montant de 538,099 livres, 7 sols et 11 deniers démontre que le roi subvient à la solde et aux frais d'entretien du régiment de Carignan-Sallières et des quatre compagnies d'infanterie des régiments de Chambellé, d'Orléans, de Poitou et de l'Allier. C'est aussi la métropole qui défraie certaines dépenses imprévues et les appointements des administrateurs supérieurs de la colonie: le marquis de Tracy, le gouverneur Courcelles, l'intendant Talon. A ces dépenses du roi, s'ajoutent les gratifications accordées aux officiers des troupes, à l'évêque de Pétrée, aux Religieuses Hospitalières de Québec et de Montréal. Des fonds sont assignés à la construction de navires et de manufactures et à l'achat de marchandises et de vivres.

Faute de documentation détaillée, il me paraît utile de grou: per les années 1667 et 1668 . Cette étude permet de vérifier l'énoncé de Guy Frégault, à savoir que dans la période antérieure à 1700 , « la documentation est trop incomplète pour qu'on puisse retracer une ligne d'évolution même grossièrement approximative ${ }^{16}$

Les dépenses relatives à l'entretien des troupes, aux appointements du gouverneur et de l'intendant, aux frais d'immigration de 200 personnes et aux gratifications s'élevaient à 242,914 livres et 15 sols en 1667 et $1668 .{ }^{17}$

Il faut inclure au montant des débours effectués lors de la première administration de Talon, les fonds expédiés à la Compagnie des Indes Occidentales. L'intendant a relevé dans un mémoire présenté à Colbert la somme des frais d'immigration que la Compagnie avait reçue du roi à partir de 1665 jusqu'à 1668 inclusivement. ${ }^{18}$

15 AC, C11A, 113-1: 12-15.

16 RHAF, op. cit. 314.

17 AC, C11A, volume 3, copie microfilmée F3 des Archives Publiques du Canada, 10.

18 RAPQ (1930 - 1931), 103 - 106. 
Talon s'appuie sur une déclaration des représentants de la Compagnie des Indes Occidentales. Les débours du roi pour les frais d'immigration de l'année 1665 s'élèvent à 52,841 livres. L'intendant note qu'en 1666 «il ne fut envoyé pour le compte du Roy ny hommes ny animaux et si la Compagnie a fait passer pour son compte les 35 personnes qu'elle marque, on l'a fait desdommager par les habitans qui ont pris ces 35 personnes à leur service ».

Pour les années 1667 et 1668 respectivement, la métropole a remis à la Compagnie des Indes Occidentales, selon Talon, les sommes de 63,340 et 33,790 livres. Le 15 juin 1668, un état des affaires de la Compagnie des Indes Occidentales, rédigé de façon imprécise, rapporte que le « Capital fourny par le Roy a diverses fois » à partir du 17 août 1664 jusqu'au 4 septembre ? (sic), probablement 1667 , se chiffre à 1,922,000 livres. ${ }^{19}$

La seconde période d'administration de Talon contient moins de précisions d'ordre financier que la première. Le chercheur doit s'accommoder des renseignements consignés dans les ordonnances au garde du trésor royal, Etienne Jehannot.

Une fois dépouillées, ces ordonnances permettent d'établir les tableaux I, II, III.

TABLEAU I

ORDONNANCES DE 1669

Date

Détail

Montant

(livre, sol, denier)

16 février 166920 Paiement tant de la dépense à faire pour la levée et le passage de 500 personnes de l'un et de l'autre sexe que de 12 cavales, 2 étalons et 50 brebis ... $\quad 64,659$

12 février 166921 Subsistance de 4 compagnies d'infanterie établies au Canada, composées chacune de 53 hommes pendant l'année 1669

26,059. 4 .

19 AC, Série F2A, carton 15, (M.G. 1/17, 2), non paginé.

20 AC, B1: 115.

21 Ibid., 123. 
22 mars $166922 \quad$ Solde et entretien de 25 soldats en chacune des 4 compagnies restées au Canada en 1669 ............................................... $\mathbf{9 , 1 5 0}$

26 mars $166923 \quad$ Fonds destinés aux officiers et aux particuliers ......................................................... 42,450

22 mars $166924 \quad$ Solde et entretien pendant les 6 premiers mois de l'année 1670 des 4 compagnies d'infanterie qui sont restées au Canada

17,580. 10

29 mars $166925 \quad$ Subsistance de 6 compagnies d'infanterie passant en Canada pendant les 6 premiers mois de 1670

19,517. 10. 8 .

\section{TABLEAU II}

ORDONNANCES DE 1670

Date

$1^{\text {er }}$ avril 167026

$1^{\text {er }}$ avril 167027

3 avril 167028

3 avril 167029

2 avril 167030

3 avril 167031

\section{Détail}

Subsistance de 6 compagnies qui doivent passer en Canada pendant les 6 derniers mois de 1670

19,571. 2 .

Subsistance de 6 compagnies qui doivent passer en Canada pendant les 6 premiers mois de 1671

19,571. 10 .

Frais d'immigration des engagés et des jeunes filles au Canada

39,138. 15 .

Appointements du gouverneur Courcelles pour l'année 1670

12,250 .

Appointements de l'intendant Talon pour l'année 1670 et les trois premiers mois de 1671

16,843. 15 .

Gages du Conseil de Talon

22 Ibid., 125.

23 Ibid., 140 - 145.

24 Ibid., 127 - 128.

25 Ibid., 131 - 132.

26 AC, B2: 40 - 41.

27 Ibid., $42-43$.

28 Ibid., 44 - 45.

29 Ibid., 46 - 47.

30 Ibid., 48 - 49.

31 Ibid., 50. 
6 avril 167032

3 avril $1670^{33}$

3 avril $1670^{34}$
Appointements de deux gardes de la prévôté de l'hôtel servant près le Sieur Talon en Canada

1,633. 7. 4 .

28,176 . «pour advances faictes pour le Canada en 1669 »

Paiement tant des appointements des charpentiers et autres ouvriers qui doivent passer en Canada pour y bâtir et construire des vaisseaux que pour la façon des bois nécessaires à ces ouvrages
30,000 .

L'état des dépenses du roi, le 11 février 1671, contient plusieurs chapitres relatifs aux gratifications des officiers, aux subventions destinées aux institutions religieuses, aux frais d'immigration. De plus, une somme de 40,000 livres est assignée à la construction navale, une somme de 10,000 livres doit être employée à l'exploitation des gisements de fer, 600 livres serviront à défrayer le coût d'établissement d'une fabrique de goudron. Cet état financier s'élève à 124,520 livres dont le montant est reporté au Tableau III.

TABLEAU III

ORDONNANCES DE 1671

Date

11 février 167135

11 février 167136

11 février 167137

11 février 167138

\section{Détail}

État des dépenses du roi

Frais d'immigration de 100 engagés et de 150 jeunes filles

Gages du Conseil de Talon

Appointements de Talon et de son secrétaire pour l'année 1671 et trois mois de 1672
Montant (livre, sol, denier) 124,520 .

39,812. 10 . 1,500 .

13,475 .

32 Ibid., 51 - 52.

33 Ibid., 53 - 54.

34 Ibid., 55.

35 AC, B3: 33 - 39.

36 Ibid., 40.

37 Ibid., 42.

38 Ibid., 43 - 44. 
11 février $1671^{39}$ Appointements du gouverneur Courcelles

12,250 .

16 février $1671^{40}$ Appointements de deux archers de la prévôté servant près le Sieur Talon ...

1,333. 7. 4 .

Le 4 juin 1672, une lettre de Louis XIV à l'intendant annonce que les dépenses militaires en Europe ne permettent pas d'accorder les mêmes assistances à la colonie que dans le passé; le même jour, Colbert écrivait: «Sa Majesté ne peut faire cette année aucune dépense pour le Canada $\gg^{41}$

Placés les uns à la suite des autres, ces états financiers, si incomplets qu'ils résistent à l'analyse statistique, nous donnent une idée très approximative des "grandes dépenses ${ }^{42} \mathrm{du}$ roi au Canada à l'époque de l'intendance de Jean Talon.

Roland Lamontagne, Ph.D., Faculté des Arts, Université de Montréal.

41 RAPQ (1930-1931), 169.

42 Ibid., 143. 